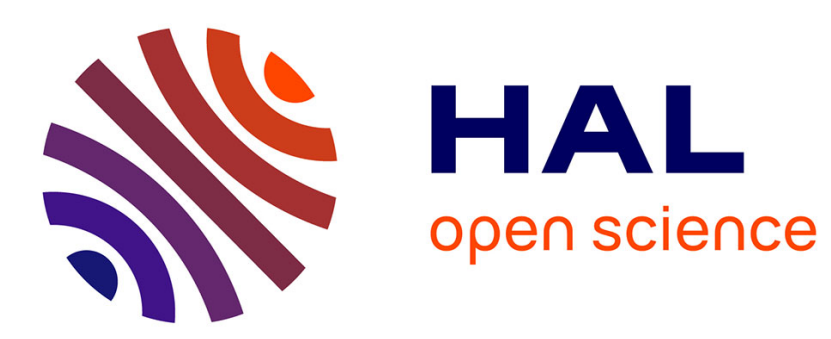

\title{
FROTTEMENT INTERNE DES VERRES DE BOROPHOSPHATES ALCALINS
}

\author{
J. Phalippou, T. Woignier, J. Zarzycki
}

\section{To cite this version:}

J. Phalippou, T. Woignier, J. Zarzycki. FROTTEMENT INTERNE DES VERRES DE BOROPHOSPHATES ALCALINS. Journal de Physique Colloques, 1983, 44 (C9), pp.C9-165-C9-170. 10.1051/jphyscol:1983920 . jpa-00223366

\section{HAL Id: jpa-00223366 https://hal.science/jpa-00223366}

Submitted on 1 Jan 1983

HAL is a multi-disciplinary open access archive for the deposit and dissemination of scientific research documents, whether they are published or not. The documents may come from teaching and research institutions in France or abroad, or from public or private research centers.
L'archive ouverte pluridisciplinaire HAL, est destinée au dépôt et à la diffusion de documents scientifiques de niveau recherche, publiés ou non, émanant des établissements d'enseignement et de recherche français ou étrangers, des laboratoires publics ou privés. 
J. Phalippou, T. Woignier et J. Zarzycki

Laboratoire de Science des Matériaux et Laboratoire des Verres du CNRS, université de Montpellier 2, 34060 Montpellier Cedex, France

Résumé - L'étude du frottement interne des verres de borates et de borophosphates alcalins a permis de mettre en evidence l'influence de $\mathrm{P}_{2} \mathrm{O}_{5}$ sur l'apparition d'un second pic de relaxation. Son intensi té dépend de la teneur initiale en eau du matériau, mais elle est aussi fortement augmentée lorsque le verre est soumis à une attaque par la vapeur d'eau. Deux hypothèses peuvent être êmises pour rendre compte de ces phénomènes.

Abstract - Internal friction studies of borates and borophosphates glasses enable us to show the influence of $\mathrm{P}_{2} \mathrm{O}_{5}$ by the appearance of a second relaxation peal on spectra. The intersity of the peak depends on the starting water content of the material and also on the reaction of the fiber with air moisture. We propose two explanations for these phenomena.

\section{I - INTRODUCTION}

Un pic de frottement interne apparaît à des températures relativement élevées dans certains verres. Pour ceux-ci, l'intensité de ce pic dépend de leur teneur en eau. Un verre mis au contact de solutions aqueuses est plus ou moins rapidement attaqué suivant sa nature et sa composition. Les verres de borates et de borophosphates ont une durabilite chimique faible et réagissent spontanëment avec la vapeur d'eau. Le présent travail a pour objet de montrer l'évolution des spectres de frottement interne de ces verres en fonction de l'attaque par la vapeur d'eau.

\section{II - TECHNIQUE EXPERIMENTALE}

Les verres ont été préparés par fusion en creuset de Platine-10 \% Rhodium, d'un mélange de carbonate de sodium, d'acide borique et de phosphate de sodium de qualité "pour analyse". Nous avons fait varier les temps d'affinage pour obtenir des verres de teneur différente en eau. Dans certains cas la fonte comprenait une quantité mineure de nitrate de sodium pour activer l'affinage.

Des fibres dont le diamètre est compris entre 0,4 et $0,8 \mathrm{~mm}$ ont été étirées à partir du liquide verrogène. Elles sont immédiatement disposées sur un pendule de torsion travaillant sous un vide primaire. La Eréquence d'oscillation varie entre 0,5 et $1 \mathrm{~Hz}$ suivant le diamètre de la fibre. Toutes les fibres ont subi un traitement de recuit identique. Le recuit est réalisé de la maniêre suivante : la fibre est portée à une température telle que la valeur du frottement interne est de $10^{-2}$. La fibre est considérée comme recuite lorsque, à une température donnée, 


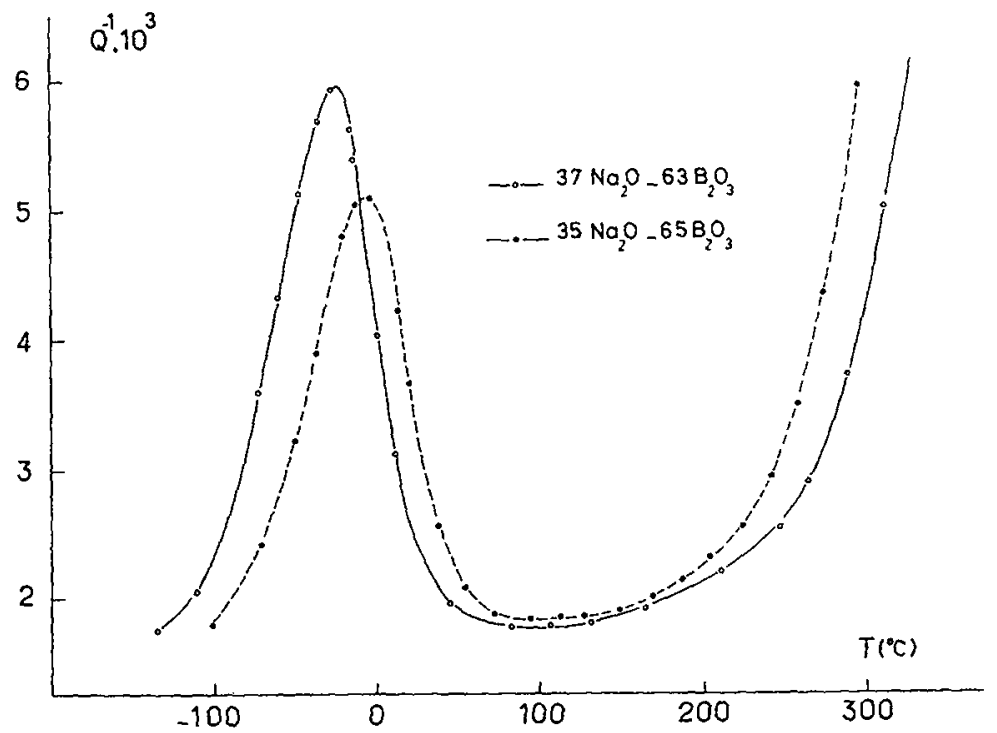

Figure 1

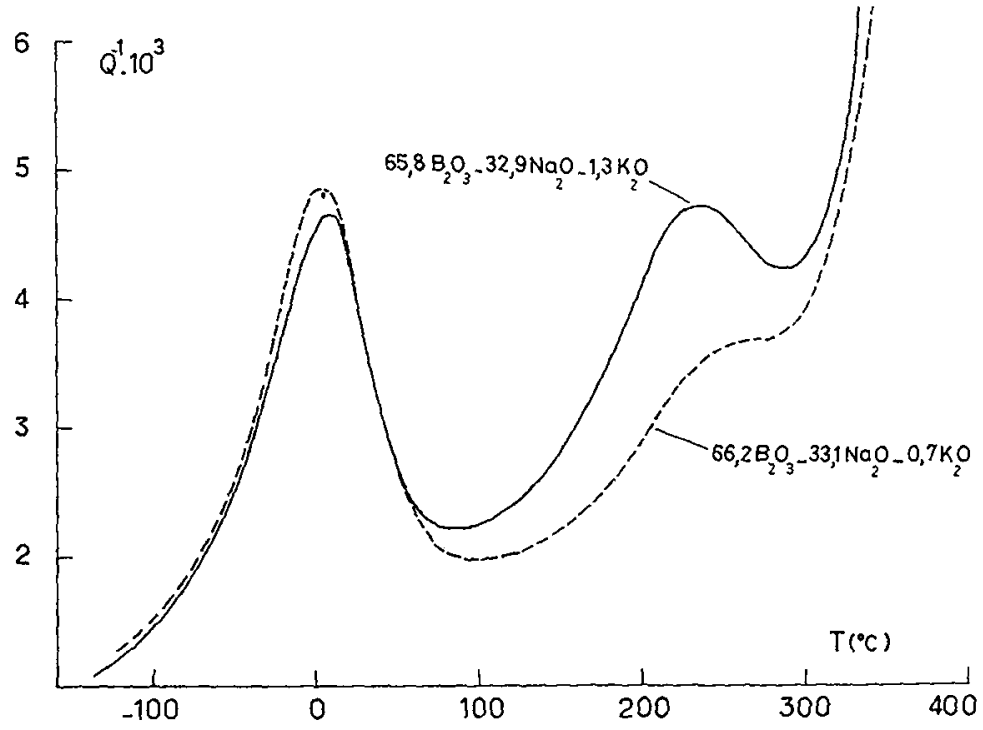

Figure 2 
la valeur du frottement interne $\left(10^{-2}\right)$ n'évolue pas dans un intervalle de temps de 15 minutes. En effet un recuit imparfait de la fibre de verre entrâne l'obtention d'un spectre de frottement interne mal résolu où le fond continu apparast à des valeurs élevées de $Q^{-1}$. La mesure du frottement interne s'effectue selon les températures décroissantes, après maintien de la fibre pendant 10 minutes à la température choisie. Elle est déduite de la mesure du décrément logarithmique de I'amplitude des oscillations.

\section{RESULTATS ET DISCUSSION}

Pour les verres de borates alcalins, le spectre de frottement interne ne présente aucun pic de relaxation à haute température (1) même pour des teneurs en oxyde alcalin très élevées (Fig.1).

Les verres de borates contenant des oxydes alcalins mixtes possèdent un second pic de frottement interne (Fig2) dû au mouvement coopératif alcalin 1 - alcalin 2 . Nous avons montré que le mouvement coopératif proton-alcalin est peu vraisemblable et que le second pic de frottement interne est probablement dû à une entité chimique du verre dans laquelle participent des liaisons hydrogènes (2). La présence de telles liaisons n'a pu être mise en évidence dans les verres de borates qui pourtant contiennent de l'eau structurale. Par ailleurs, on a pu déceler la présence d'eau moléculaire dans divers verres (3).

Les études actuelles montrent que l'eau moléculaire joue un grand rôle dans l'attaque des verres de silicates. C'est ainsi que cette espèce chimique réagirait sur les atomes d'oxygènes non pontants liés aux ions alcalins du verre pour former des groupes silanols (4). La neutralisation de la charge électrique rend l'ion alcalin relativement mobile. Il se lie alors au contre-ion $\mathrm{OH}^{-}$. Un modèle de l'entité structurale responsable du second pic de frottement interne est présenté ci-dessous. Les cations $\mathrm{Na}^{+}$se présentent sous forme de paires, comme les mesures de RMN 1 'ont mis en évidence (5). On peut envisager un environnement tétraédrique de I'atome d'oxygène de la molécule d'eau(6) qui est essentielle au transport des espèces chimiques sous I'effet de la contrainte.

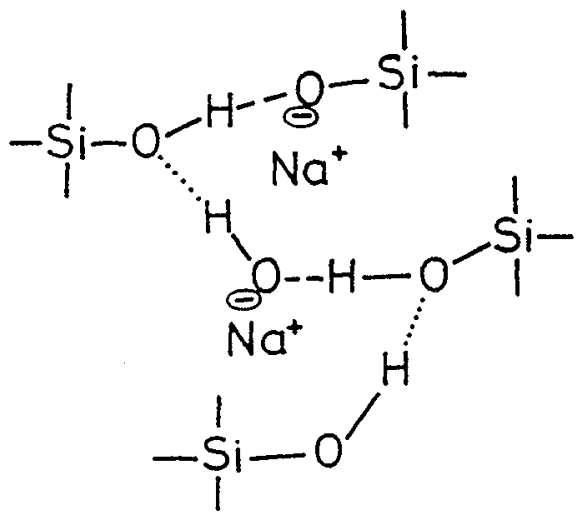




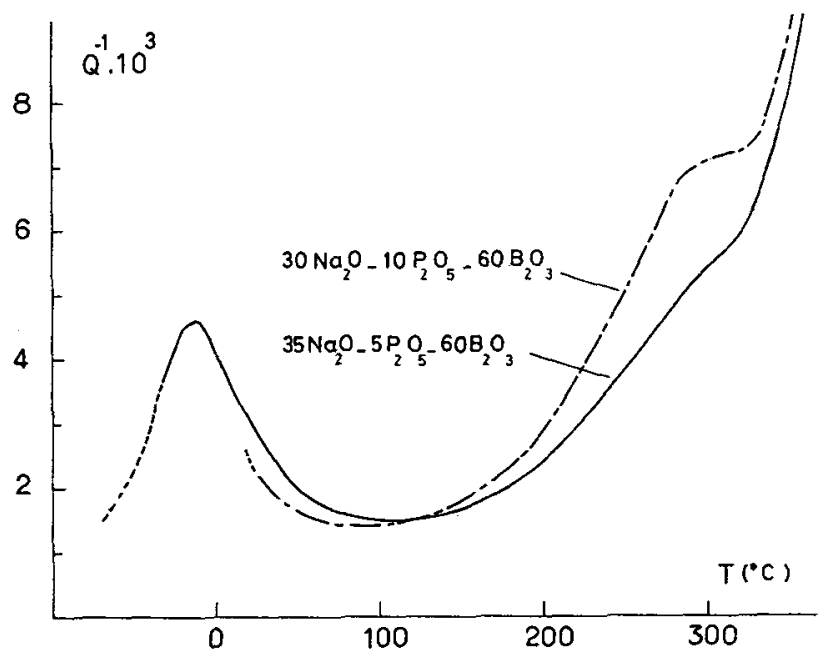

Figure 3
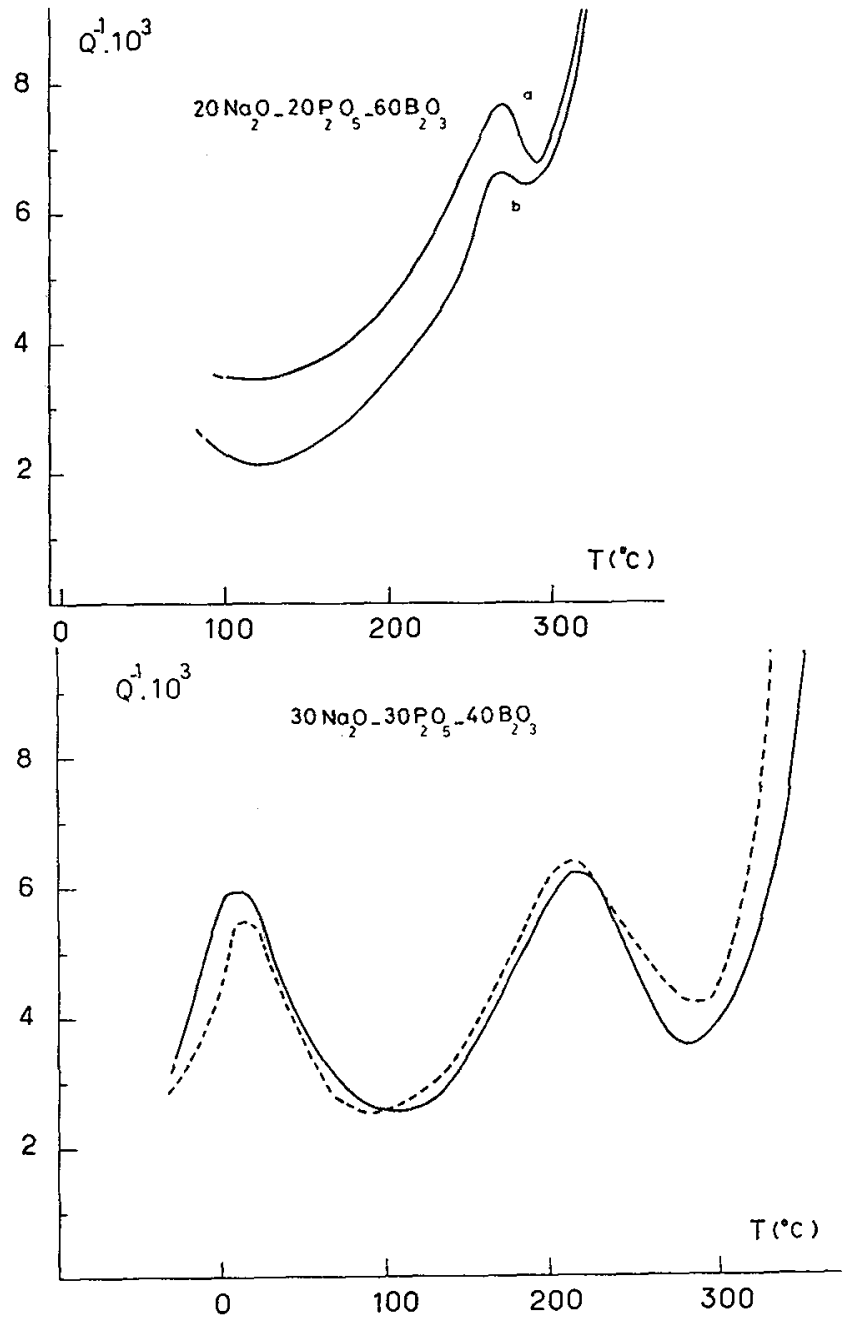

Figure 5

Figure 4 
Lorsque I'on fait réagir une fibre de verre de silicate alcalin avec la vapeur d'eau, le spectre de frottement interne en est affecté (7) et le pic haute température augmente en intensité(8). Cette augmentation est analogue à celle constatée lorsque le verre initial présente des teneurs en eau croissantes. Il apparâ̂t donc que le second pic de frottement interne est indépendant de la forme sous laquelle l'eau est introduite dans la fibre dans le cas des verres de silicates.

Quelle que soit leur teneur en eau, les verres de borates alcalins ne présentent aucun second pic sur leur spectre de frottement interne. Cependant 1 'addition de $\mathrm{P}_{2} \mathrm{O}_{5}$ le fait apparâtre. Un épaulement du côté de la remontée du fond continu est visible sur la figure 3 pour un verre contenant 5o molaire d'anhydride phosphorique. Cet épaulement se développe pour donner un pic bien défini lorsque la teneur en $\mathrm{P}_{2} \mathrm{O}_{5}$ est de $20 \%$ molaire ( $\mathrm{Fig.4}$ courbe b). La même fibre maintenue à température ambiante, dans une atmosphère saturée d'eau possède un spectre de frottement interne dans lequel le second pic a augmenté en intensité (Fig.4 courbe a). On notera aussi que le fond continu s'établit à des valeurs plus élevées, ce qui dénote l'augmentation conséquente du second pic qui s'étend alors sur un domaine de température plus grand et de ce fait vient aux basses températures interférer avec le premiex pic dû à la diffusion sous contrainte des ions alcalins (non montré sur la figure).

Le second pic de frottement interne est particulièrement bien résolu dans les verres de borophosphates de sodium de composition $20 \mathrm{Na} 2 \mathrm{O}-30 \quad \mathrm{P}_{2} \mathrm{O}_{5}-4 \mathrm{~B}_{2} \mathrm{O}_{3}$. La courbe en pointillé correspond au verre affiné pendant une heure, celle en trait plein au verre affiné pendant 3 heures. Les spectres sont sensiblement identiques. Le temps d'affinage n'influe que très peu sur la courbe de frottement interne. L'attaque de la fibre par 1'eau atmosphérique semble être beaucoup plus efficace. Ceci peut provenir soit du fait que les verres de borophosphates réagissent fortement avec l'eau atmosphérique, soit que la quantité d'eau dissoute ne diminue pas considérablement en fonction du temps de maintien à haute température du liquide verrogène.

Après attaque par l'eau atmosphérique ou dans une atmosphère saturée d'eau les fibres se recouvrent d'une pellicule blanchâtre qui n'a pû être pour l'instant analysée. Il s'agit probablement de carbonate de sodium et d'acide borique. Il s'en suit que la fibre elle-même se trouve enrichie en $\mathrm{P}_{2} \mathrm{O} 5$, et de ce fait, la composition du verre réellement analysé peut être voisine de celle d'un verre dont le second pic de frottement interne est plus développé.

La variation de composition s'effectuerait en surface de la fibre et le coeur aurait une composition inchangée. I.e profil de concentration des ions alcalins a été étudié par SMETS et IOMMEN dans les verres de silicates exposés à une solution aqueuse à $70^{\circ} \mathrm{C}$ (4). Le changement de composition ne s'établit que sur quelques microns. En conséquence, une autre hypothèse doit être retenue pour expliquer $I^{\prime}$ augmentation du second pic de frottement interne par attaque d'une fibre par la vapeur d'eau. L'eau entrerait dans la structure du verre sous forme d'eau moléculaire. cette espèce peut participer à l'entité chimique responsable du second pic. Avec l'augmentation de l'eau moléculaire le second pic de frottement interne s'accroit. 


\section{CONCLUSION}

Les verres de borates alcalins ont des spectres de frottement interne dépourvus de second pic. Celui-ci n'apparaît pas lorsqu' augmente leur teneur en eau. Par contre, les verres de borophosphates présentent un second pic de frottement interne. Son intensité augmente lorsque la fibre est exposée dans une atmosphère contenant de la vapeur d'eau. Elle paraît moins dépendre de la durée de l'affinage du verre.

La variation de $I$ 'intensité du second pic de frottement interne peut provenir soit d'un changement de composition du verre soit de l'augmentation de la teneur en eau moléculaire. Cette espèce chimique ferait partie de l'entité structurale responsable du second pic de frottement interne.

V. REFERENCES

(1) PHALIPPOU J., JABRA R., ZARZYCKI J., Rev.Phys.Appl.15, $(1980), 1579$.

(2) PHALIPPOU J., JABRA R., ZARZYCKI J., J.NOn Cryst.Solids $38-39,(1980), 481$.

(3) ERNSBERGER F.M., Phys.Chem.Glasses, 21, (1980), 146.

(4) SMETS B.M.J., LOMMEN T.P.A., Phys.Chem.Glasses., 23, (1969), 174 .

(5) MULLER-WARMUTH W., SCHULZ G.W., NEUROTH N., MEYER F., DEEG.E., Z.Naturforsch.2Oa (1965), 902 .

(6) WOLTERS D.R., VERMEIL J., Phys.Chem.Glasses, 22 (3), (1981), 55.

(7) VAUGIN L., BRETON J.C., GOBIN P., Verres Réfract., $23(1969), 174$.

(8) ZDANOEWSKI W.A., RINDONE G.E., Amer.Ceram.Soc.C., (1981), 183. 